\title{
Evaluation of the Role of Himpyrin Liquid in the Management of Inflammation and Pain in Cats
}

\author{
Rohith K Jayaprakash ${ }^{1}$, Vishwanath G Bhagwat ${ }^{2 *}$, Tattimani Santosh ${ }^{2}$ and Rangesh Paramesh ${ }^{2}$ \\ ${ }^{1}$ VetMedix, \#377, $9^{\text {th }}$ Main, MSR Enclave, Nagasandra Post, Bengaluru, INDIA \\ ${ }^{2}$ The Himalaya Drug Company, Makali, Bengaluru, Karnataka, INDIA \\ "Corresponding author:VG Bhagwat; E-mail:dr.bhagwat@himalayawellness.com
}

Received: 11 June, 2021

Revised: 16 July, 2021

Accepted: 21 July, 2021

\begin{abstract}
The present study was designed to evaluate in vivo efficacy of polyherbal formulation, Himpyrin liquid, for anti-inflammatory, analgesic, and antipyretic activities in cats. Twenty cats with a history of pain, inflammation, and fever due to scratch injuries/ accidents were selected $(\mathrm{G} 1 ; n=20)$ and supplemented with Himpyrin liquid along with standard treatment until complete recovery. The changes in assessment parameters score, viz. pain, fever, and activity level scores, along with adverse effects and product performance/satisfaction scores were evaluated. The results of the present study revealed that pain $(p<0.01)$ and fever $(p<0.001)$ were significantly decreased in cats as early as days 2 and 4 , respectively, following Himpyrin liquid supplementation along with standard treatment. The administration of Himpyrin liquid to cats restored the activity level of cats affected with fever and pain due to scratch injuries/accidents. Furthermore, Himpyrin liquid was considered highly satisfactory for inflammation amelioration due to scratch injuries/accidents in cats without any adverse effects. In conclusion, this study supplies considerable preliminary data to hint that Himpyrin liquid possesses anti-inflammatory activities. Hence, Himpyrin liquid at $1 \mathrm{~mL}$ twice daily along with standard treatment could be recommended for inflammation amelioration and pain in cats. However, further clinical studies evaluating biochemical parameters were recommended to be carried out to better elucidate the mechanism of action and ultimate impact on the overall health of cats.
\end{abstract}

\section{HIGHLIGHTS}

(0 Himpyrin administration alleviates inflammation and pain in cats and feline.

o Himpyrin liquid possesses anti-inflammatory activities.

Keywords: Himpyrin liquid, Cats, Inflammation, Pain, and Polyherbal formulation

Pain in cats has many physiological and emotional negative effects (Hellyer et al., 2007). Pain delays recovery, impacts negatively on a patient's wellbeing, and disturbs the bond with its owner and also the veterinary team (Epstein et al., 2015; Teixeira et al., 2013). Signs of pain may be subtle and include withdrawing from attention, decreasing mobility, reducing interactions with humans and other animals, poor appetite, and aggression. Chronic pain is pain that has persisted for more than 2-3 weeks, often persists months or years, and may continue beyond the anticipated healing time. Importantly, chronic pain can become dissociated from the inciting cause and be maladaptive, such that the degree of pain does not necessarily correlate with the pathology observed or perceived by the individual, and is not associated with healing (Gowan et al., 2011).

Studies have looked at the use of nonsteroidal antiinflammatory drugs (NSAIDs) for acute, especially perioperative pain in cats (Benito et al., 2016; Steagall and Monteiro-Steagall, 2013; Mush et al., 2014). Multimodal

How to cite this article: Jayaprakash, R.K., Bhagwat, V.G., Santosh, T. and Paramesh, R. (2021). Evaluation of the Role of Himpyrin Liquid in the Management of Inflammation and Pain in Cats. J. Anim. Res., 11(04) 701-705.

Source of Support: None; Conflict of Interest: None 
analgesia is commonly advocated, but NSAIDs playing a key role in managing chronic feline pain, especially musculoskeletal pain, is becoming evident just as they do in humans and dogs (Gowan et al., 2011; Guillot et al., 2013). Although many NSAIDs have been available to treat dogs with degenerative joint disease (Bhathal et al., 2017) only a restricted range has been licensed for shortterm (up to a few days) use in cats. Meloxicam, an NSAID, has been licensed for long-term use in cats in many regions of the world, transforming the ability to manage pain in this species, and robenacoxib, another NSAID, has been licensed for up to 6 days of therapy in cats (Gowan et al., 2011; Guillot et al., 2013). However, adverse drug events related to the use of NSAID most commonly affect the gastrointestinal system, liver, kidneys, and platelet function (Bhathal et al., 2017). While the need for and benefit of NSAID therapy in many situations is clear, screening and monitoring are important for the clinician, owner, and patient to help minimize the likelihood of occurring adverse effects (Ziegler et al., 2017).

Persistent long-term use safety concerns must be considered when prescribing these NSAIDs medications for chronic and degenerative pain conditions. Although nonsteroidal medications can be effective, herbs and dietary supplements may offer a safer and often an effective alternative treatment for pain relief, especially for longterm use. Maroon et al. reported the potentially serious side effects of nonsteroidal drugs and commonly used and clinically studied natural alternative anti-inflammatory supplements viz. Curcuma longa, green tea, Pycnogenol derived from Pinus maritima, Boswellia serrata resin, resveratrol derived from Polygonum cuspidatum, Uncaria tomentosa, Uncaria guianensis, and Capsicum annum (Ambriz-Perez et al., 2016). Also literature study shows the anti-inflammatory activities of herbs namely Zingiber officinale (Shin et al., 2015). Cyperus rotundus (Musta) (Ahmad et al., 2014) Acorus calamus (Vacha) (Phanse et al., 2012) and Tinospora cordifolia (Guduchi) (Philip et al., 2018).

With this scenario and the growing acceptance of traditional herbal preparations, a polyherbal formulation, Himpyrin liquid, was developed by The Himalaya Drug Company. Moreover, the Himpyrin liquid is claimed to possess anti-inflammatory activities in felines. Hence, in vivo efficacy of Himpyrin liquid was assessed in cats in the present study to verify its role in the management of inflammation and pain in cats and felines.

\section{MATERIALS AND METHODS}

\section{Polyherbal formulation}

Himpyrin liquid is a proprietary polyherbal formulation developed by The Himalaya Drug Company, Bengaluru, India, composed of $Z$. officinale, $C$. rotundus, $A$. calamus, G. glabra, and T. cordifolia.

\section{Ethical committee approval}

The present study was conducted following the guidelines laid down for the care and use of animals. Moreover, the study protocol was approved by the Institutional Animal Ethics committee, The Himalaya Drug Company, Bangalore (protocol no. AHP/SA/10/18).

\section{Study subjects}

Twenty cats with a history of pain, inflammation, and fever due to scratch injuries/accidents were presented at the Vet Medix Clinic, Bengaluru, Karnataka, and enrolled in the current study. The study details, treatment plan, outcomes, and other pros and consequences were explained to the pet owner. In addition, consent was obtained before study enrollment.

\section{Study design and experimental details}

Twenty cats with a history of pain, inflammation, and fever due to scratch injuries/accidents were selected (G1; $n=20$ ) and supplemented with Himpyrin liquid along with standard treatment until complete recovery. Cats were administered with subcutaneous Convenia injection at a dose of $3.6 \mathrm{mg} / \mathrm{lb}(8 \mathrm{mg} / \mathrm{kg})$ body weight based on the severity of pain and fever. Concurrently, crusts due to injury on the skin were gently removed with a brush and cleaned with sterile saline solution and wiped with dry sterile cotton. Concurrent treatment with other analgesics, antipyretic, and anti-inflammatory supplements was discontinued when Himpyrin liquid is being administered to the cats. 


\section{Evaluation of study parameters}

The changes in assessment parameters score, viz. pain, fever, and activity level scores, along with adverse effects and product performance/satisfaction scores were evaluated following Himpyrin liquid supplementation to access the role of Himpyrin liquid in the management of inflammatory conditions in cats according to the grading system (Taraphdar et al., 2018) described in Table 1.

Table 1: Assessment Parameters Grading System

\begin{tabular}{|c|c|c|}
\hline Parameter & Description & Score \\
\hline \multirow[t]{2}{*}{ Fever } & $\begin{array}{l}\text { Normal body temperature, } 100.4^{\circ} \mathrm{F} \text { to } \\
102.5^{\circ} \mathrm{F}\end{array}$ & 0 \\
\hline & Abnormal body temperature, $\geq 102.5^{\circ} \mathrm{F}$ & 1 \\
\hline \multirow{4}{*}{ Pain } & $\begin{array}{l}\text { Normal: No pain, no overt signs of } \\
\text { discomfort, and no resentment to firm } \\
\text { pressure }\end{array}$ & 0 \\
\hline & $\begin{array}{l}\text { Mild pain: No overt signs of discomfort } \\
\text { but resentment to firm pressure }\end{array}$ & 1 \\
\hline & $\begin{array}{l}\text { Moderate pain: Some overt signs of } \\
\text { discomfort which are made worse by firm } \\
\text { pressure }\end{array}$ & 2 \\
\hline & $\begin{array}{l}\text { Severe pain: Obvious sign of persistent } \\
\text { discomfort which are made worse by firm } \\
\text { pressure }\end{array}$ & 3 \\
\hline \multirow{3}{*}{ Activity level } & Normal: Active and alert & 0 \\
\hline & Dull and depressed & 1 \\
\hline & Sluggish and lethargy & 2 \\
\hline \multirow{2}{*}{ Activity level } & Absent & 0 \\
\hline & Present & 1 \\
\hline \multirow{4}{*}{$\begin{array}{l}\text { Product } \\
\text { performance/ } \\
\text { Satisfaction } \\
\text { score }\end{array}$} & Highly satisfied & 4 \\
\hline & Moderately satisfied & 3 \\
\hline & Neither satisfied nor dissatisfied & 2 \\
\hline & Not satisfied (no relief) & 1 \\
\hline
\end{tabular}

\section{Statistical analysis}

The data are expressed as mean \pm standard error of the mean (SEM). Data were subjected to a two-way analysis of variance followed by the Bonferroni test to draw the comparison between day 1 and the remaining treatment days. In addition, $p \leq 0.05$ was considered statistically significant.

\section{RESULTS AND DISCUSSION}

Pain $(p<0.01)$ and fever $(p<0.001)$ was significantly decreased in cats as early as days 2 and 4 , respectively, following Himpyrin liquid supplementation along with standard treatment. However, complete alleviation of pain and fever was observed on day 5 . The activity level score (mean \pm SEM) exhibited by animals on day 1 was $1.50 \pm$ 0.18 . However, animals showed a statistically significant $(p<0.001)$ improvement in activity level score as early as day 2 onwards compared with day 1 following Himpyrin liquid supplementation along with standard treatment and restored to normal $(0.00 \pm 0.00)$ on day 5 . The activity level score revealed that 5 days of Himpyrin liquid supplementation along with standard treatment is required for complete restoration of the activity level of cats with fever and pain due to scratch injuries/accidents (Table 2). The adverse effects and product performance/satisfaction scores of Himpyrin liquid were $0.00 \pm 0.00$ and $3.75 \pm$ 0.10 , respectively (Table 3 ).

Thesefindings depicted that Himpyrinliquid was considered highly satisfactory for inflammation amelioration due to scratch injuries/accidents in cats. These findings could be attributed to the anti-inflammatory activities of Himpyrin liquid. Furthermore, Himpyrin liquid supplementation in cats has no adverse effects indicating that ingredients used in the products are safe for oral supplementation in cats.

The anti-inflammatory activities of Himpyrin liquid could be understood by individual herbal ingredients, viz. $Z$. officinale, C. rotundus, A. calamus, G. glabra, and $T$. cordifolia present in the Himpyrin liquid. Moreover, Hassan et al. reported that gingerol, shogaol, and other structurally related substances in ginger inhibit prostaglandin and leukotriene biosynthesis through suppression of 5-lipoxygenase or prostaglandin synthetase. Additionally, they can also inhibit the synthesis of proinflammatory cytokines (e.g., interleukin (IL)-1, tumor necrosis factor- $\alpha$ (TNF- $\alpha$ ), and IL-8) (Rahmani, 2014; Jafarnejad et al., 2017). The paw edema in carrageenan-induced rats was considerably reduced by treating with $400 \mathrm{mg} / \mathrm{kg}$ aqueous ginger extracts when compared with untreated rats $(p<$ $0.001)$. Hence, the investigations of Hassan et al. showed that the aqueous extract of $Z$. officinale possesses antiinflammatory properties (Hassan, et al., 2017).

Fulgidic acid from the rhizomes of $C$. rotundus and the underlying mechanisms involved in its anti-inflammatory activity was evaluated by Shin et al. (2015) The results revealed that fulgidic acid reduced the production of nitric oxide, prostaglandin E2, TNF- $\alpha$, and IL-6 
Table 2: Effect of Himpyrin Liquid on Assessment Parameters in Cats

\begin{tabular}{llllll}
\hline Parameter & Day 1 & Day 2 & Day 3 & Day 4 & Day 5 \\
\hline Fever score & $0.85 \pm 0.08$ & $0.85 \pm 0.08$ & $0.60 \pm 0.11$ & $* * * 0.20 \pm 0.09$ & $* * * 0.00 \pm 0.00$ \\
Pain score & $2.25 \pm 0.24$ & $* * 1.80 \pm 0.25$ & $* * * 1.10 \pm 0.18$ & $* * * 0.35 \pm 0.11$ & $* * * 0.00 \pm 0.00$ \\
Activity level score & $1.50 \pm 0.18$ & $* * * 1.00 \pm 0.16$ & $* * * 0.70 \pm 0.11$ & $* * * 0.25 \pm 0.10$ & $* * * 0.00 \pm 0.00$ \\
\hline
\end{tabular}

Values are expressed as mean $\pm \mathrm{SEM} ; n=20 ;{ }^{* *} p<0.01$ and ${ }^{* * *} p<0.001$ compared with day 1 based on repeated-measures two-way analysis of variance followed by the Bonferroni test.

Table 3: Effect of Himpyrin Liquid on Adverse Effect and Product Performance Score in Cats

\begin{tabular}{ll}
\hline Parameter & Score \\
\hline Adverse effects & $0.00 \pm 0.00$ \\
Product performance/product satisfaction & $3.75 \pm 0.10$ \\
\hline
\end{tabular}

Values are expressed as mean $\pm \mathrm{SEM} ; n=20$.

in lipopolysaccharide (LPS)-induced RAW264.7 macrophages. In addition, fulgidic acid suppressed the LPS-induced expression of inducible nitric oxide synthase (iNOS) and cyclooxygenase-2 (COX-2) at the protein level, as well as iNOS, COX-2, TNF- $\alpha$, and IL-6 at mRNA levels. Furthermore, fulgidic acid suppressed the LPS-induced transcriptional activity of activator protein-1 (AP-1) as well as the phosphorylation of c-Fos and c-Jun. In addition, fulgidic acid did not show any effect on LPSinduced nuclear factor $\kappa \mathrm{B}$ activity. In summary, these results suggest that the anti-inflammatory effect of fulgidic acid was associated with the suppression of iNOS, COX2 , TNF- $\alpha$, and IL- 6 expressions through downregulating AP-1 activation in LPS-induced RAW264.7 macrophages (Shin et al., 2015). In another study reported by Ahmad et al., (2014) the crude extract of C. rotundus was analyzed for anti-inflammatory activity. The results revealed that at 300 and $500 \mathrm{mg} / \mathrm{kg}$ doses of crude extract C. rotundus was more effective at 2 and $4 \mathrm{~h}$, respectively. The maximum percentage of inhibition (36\%) observed with a $500 \mathrm{mg} / \mathrm{kg}$ dose. The findings of this study showed that protection from edema and pain was evident following supplementation of crude extract of C. rotundus (Ahmad et al., 2014).

A preclinical study evaluating the anti-inflammatory activity of the chloroform extract of $T$. cordifolia (Willd.) conducted by Philip et al. reported that the LPSinduced upregulation of proinflammatory biomarkers was significantly inhibited by the chloroform extract of T. cordifolia without inhibiting COX-1. The chloroform extract of T. cordifolia and LPS-incubated cells showed reduced phosphorylated p38 MAPK levels, and higher levels of NF- $\kappa \mathrm{B}$ were retained in the cytoplasm. Furthermore, rats pretreated with chloroform extract of T. cordifolia showed a statistically significant decrease in paw edema ( $p \leq 0.05)$ (Philip et al., 2018).

A review published by Phanse et al. (2012) reported that $A$. calamus leaf extract inhibits the production of proinflammatory cytokines through multiple mechanisms and could be considered as a novel and effective antiinflammatory agent (Phanse et al., 2012). Furthermore, another study evaluating the anti-inflammatory activity of $80 \%$ ethanolic extract of $A$. calamus Linn. leaves in albino rats using the carrageenan-induced paw edema and cotton pellet granuloma tests revealed that $80 \%$ ethanolic extract of $A$. calamus at the dose levels of 100 and $200 \mathrm{mg} / \mathrm{kg}$ caused a significant $(p<0.05)$ reduction in the paw edema in rats. These findings delineated that the $80 \%$ ethanolic extract of $A$. calamus leaves possesses anti-inflammatory activity probably through the reduction of various biochemicals, viz. histamine, 5-HT, and various kinases which are involved in the early inflammation phases (Jain et al., 2010).

\section{CONCLUSION}

In conclusion, this study delineated that cats and feline suffering from inflammation and pain due to scratch injuries/accidents were ameliorated following Himpyrin liquid administration. Hence, this supplies considerable preliminary data to hint that Himpyrin liquid possesses 
anti-inflammatory activities. However, further clinical study evaluating biochemical parameters is recommended to better elucidate the mechanism of action and ultimate impact on the overall health of cats.

\section{ACKNOWLEDGMENTS}

The authors expressed their deep gratitude to Dr. Rajesh Kumawat and Dr. U.V. Babu for their encouragement and support for the study.

\section{REFERENCES}

Ahmad, M., Rookh, M., Rehman, A.B., Muhammad, N., Younus, M. and Wazir, A. 2014 Assessment of anti-inflammatory, anti-ulcer and neuro-pharmacological activities of Cyperus rotundus Linn. Pak. J. Pharm. Sci., 27(6 Spec No.): 22412246

Ambriz-Pérez, D.L., Leyva-López, N., Gutierrez-Grijalva, E.P. and Heredia, J.B. 2016. Phenolic compounds: Natural alternative in inflammation treatment. A Review. Cogent Food Agric., 2(1): 1131412.

Benito, J., Monteiro, B., Lavoie, A.M., Beauchamp, G., Lascelles, B.D.X. and Steagall, P.V. 2016. Analgesic efficacy of intraperitoneal administration of bupivacaine in cats. $J$. Feline Med. Surg., 18(11): 906-912.

Bhathal, A., Spryszak, M., Louizos, C. and Frankel, G. 2017. Glucosamine and chondroitin use in canines for osteoarthritis: A review. Open Vet. J., 7(1): 36-49.

Epstein, M., Rodan, I., Griffenhagen, G., Kadrlik, J., Petty, M., Robertson, S. and Simpson, W. 2015. 2015 AAHA/AAFP pain management guidelines for dogs and cats. J. Am. Anim. Hosp. Assoc., 51(2): 67-84.

Gowan, R.A., Lingard, A.E., Johnston, L., Stansen, W., Brown, S.A. and Malik, R. 2011. Retrospective case control study of the effects of long-term dosing with meloxicam on renal function in aged cats with degenerative joint disease. $J$. Feline Med. Surg., 13(10): 752-761.

Guillot, M., Moreau, M., Heit, M., Martel-Pelletier, J., Pelletier, J.P. and Troncy, E. 2013. Characterization of osteoarthritis in cats and meloxicam efficacy using objective chronic pain evaluation tools. Vet. J., 196(3): 360-367.

Hassan, N.A., Karunakaran, R. and Uma, S.A. 2017. Antiinflammatory effect of Zingiber officinale on Sprague Dawley rats. Asian. J. Pharm. Clin. Res., 10(3): 1-3.

Jafarnejad, S., Keshavarz, S.A., Mahbubi, S., Saremi, S., Arab, A., Abbasi, S. and Djafarian, K. 2017. Effect of ginger (Zingiber officinale) on blood glucose and lipid concentrations in diabetic and hyperlipidemic subjects: A meta-analysis of randomized controlled trials. J. Functional Foods, 29: 127-134.

Jain, D.K., Gupta, S., Jain, R. and Jain, N. July-September 2010. Anti-inflammatory activity of $80 \%$ ethanolic extract of Acorus calamus Linn. Leaves in albino rats. Res. J. Pharm. Tech., 3(3).

Musk, G.C., Murdoch, F.R., Tuke, J., Kemp, M.W., Dixon, M.J. and Taylor, P.M. 2014. Thermal and mechanical nociceptive threshold testing in pregnant sheep. Vet. Anaesth. Analg., 41(3): 305-311.

Phanse, M.A., Patil, M.J., Abbulu, Konde, Chaudhari, P.D. and Patel, B. 2012. In-vivo and in-vitro screening of medicinal plants for their anti-inflammatory activity: an overview. $J$. App. Pharm. Sci., 02 (07): 19-33.

Philip, S., Tom, G. and Vasumathi, A. V. 2018. Evaluation of the anti-inflammatory activity of Tinospora cordifolia (Willd.) Miers chloroform extract - a preclinical study. J. Pharm. Pharmacol., 70(8): 1113-1125.

Rahmani, A. H. 2014. Active ingredients of ginger as potential candidates in the prevention and treatment of diseases via modulation of biological activities. Int. J. Physiol. Pathophysiol. Pharmaco., 6(2): 125.

Shin, J.S., Hong, Y., Lee, H.H., Ryu, B., Cho, Y.W., Kim, N.J., Jang, D.S. and Lee, K.T. 2015. Fulgidic acid isolated from the rhizomes of Cyperus rotundus suppresses LPSinduced iNOS, COX-2, TNF- $\alpha$, and IL- 6 expression by AP-1 inactivation in RAW264.7 macrophages. Biol. Pharm. Bull., 38 (7): 1081-1086.

Steagall, P.V. and Monteiro-Steagall, B.P. 2013. Multimodal analgesia for perioperative pain in three cats. J. Feline Med. Surgery, 15(8): 737-743.

Taraphdar, A. K., Mukherjee, A. and Gupta, M. 2018. Antipyretic effect of a polyherbal ayurvedic formulation: A randomized controlled clinical study. J. Phytopharmacol., 7(3): 325-333.

Teixeira, R.C., Monteiro, E.R., Campagnol, D., Coelho, K., Bressan, T.F. and Monteiro, B.S. 2013. Effects of tramadol alone, in combination with meloxicam or dipyrone, on postoperative pain and the analgesic requirement in dogs undergoing unilateral mastectomy with or without ovariohysterectomy. Vet. Anaesth. Analg., 40(6): 641-649.

Ziegler, A., Fogle, C. and Blikslager, A. 2017. Update on the use of cyclooxygenase-2-selective nonsteroidal antiinflammatory drugs in horses. J. Am. Anim. Hosp. Assoc., 250(11): 1271-1274. 
